\title{
Estudo da romifidina ou xilazina associadas à cetamina em cães pré-tratados com atropina, submetidos ou não à ovario-histerectomia
}

\section{Romifidine or xylazine combined with ketamine in dogs premedicated with atropine and submitted to ovariohysterectomy}

\author{
Stélio Pacca Loureiro Luna, * Mariângela Louzano Cruz, Adriano Bonfim Carregaro, Edivaldo Marques Junior
}

\begin{abstract}
Resumo
Estudou-se o efeito cardiorrespiratório das associações de romifidina ou xilazina e cetamina em 12 cães clinicamente sadios. Seis animais receberam como medicação pré-anestésica (MPA) 0,05 mg/kg de atropina por via subcutânea (SC), seguido da associação de $0,1 \mathrm{mg} / \mathrm{kg}$ de romifidina e $15 \mathrm{mg} / \mathrm{kg}$ de cetamina por via intramuscular - ARC (IM) e seis animais receberam 0,05 $\mathrm{mg} / \mathrm{kg}$ de atropina SC, seguido de $1 \mathrm{mg} / \mathrm{kg}$ de xilazina e $15 \mathrm{mg} / \mathrm{kg}$ de cetamina IM - AXC. Os parâmetros cardiorrespiratórios foram avaliados antes da MPA e a cada 15 minutos por 120 minutos. Os protocolos anestésicos produziram hipotermia e hipercapnia com conseqüente acidose respiratória. Nenhuma alteração foi observada na freqüência cardíaca, oximetria, concentração de $\mathrm{CO}_{2}$ expirado, bicarbonato, osmolaridade sangüínea, concentração sangüínea de sódio e potássio, glicose e hematócrito. A PaO, reduziu apenas nos animais tratados com ARC aos 45 minutos. Observaram-se maiores valores de cálcio e menores de $\mathrm{pH}$ aos 45 minutos, bradipnéia e menores valores do volume aos 105 minutos e de pressão arterial aos 15 e 30 minutos nos animais tratados com ARC em relação aos tratados com AXC. Apesar das associações de ARC e AXC produzirem estabilidade dos parâmetros cardiorrespiratórios, não houve analgesia e relaxamento muscular suficientes, já que quatro animais de cada grupo necessitaram complementação anestésica com cetamina e destes, dois do grupo AXC necessitaram complementação com anestesia volátil, bem como recuperação prolongada, o que inviabiliza o uso destas técnicas anestésicas nestas doses para ovario-histerectomia em cães.
\end{abstract}

Palavras-chave: romifidina, xilazina, atropina, cetamina, cão, ovario-histerectomia.

\begin{abstract}
The cardiorespiratory and analgesic effects of romifidine or xylazine combined with ketamine was investigated in dogs. Dogs were premedicated with $0.05 \mathrm{mg} / \mathrm{kg}$ of atropine subcutaneously (SC), followed by $0.1 \mathrm{mg} / \mathrm{kg}$ of romifidine (ARK - $\mathrm{n}=6$ ) or $1.0 \mathrm{mg} /$ $\mathrm{kg}$ of xylazine $(A X K-\mathrm{n}=6)$ and $15 \mathrm{mg} / \mathrm{kg}$ of ketamine IM. Dogs of both groups developed hypothermia, hypercapnia and respiratory acidosis. There were no changes in heart rate, hemoglobin $\mathrm{O}_{2}$ saturation, expired $\mathrm{CO}_{2}$, bicarbonate, hematocrit, blood osmolarity and blood biochemistry, when compared to the control values in each group. $\mathrm{PaO}_{2}$ reduced only in dogs treated with $\mathrm{AXK}$ at 45 minutes. The $\mathrm{pH}$ was lower at $45 \mathrm{~min}$, the respiratory rate and minute volume were lower at 105 min and the mean arterial blood pressure was lower at 15 and 30min in dogs treated with ARK when compared to AXK. Although the use of ARK and AXK produced cardiorespiratory stability, the analgesia and muscle relaxation was not adequate for ovariohysterectomy and recovery was prolonged.
\end{abstract}

Keywords: romifidine, xylazine, atropine, ketamine, dogs, ovariohysterectomy.

\section{Introdução}

A anestesia geral inalatória apresenta algumas vantagens, entre elas, a rapidez de ação, menor risco de sobredose, adequação do plano anestésico de acordo com o tipo de cirurgia, possibilidade de controle do tempo hábil e maior segurança cardiorrespiratória. Por outro lado, algumas desvantagens como a contaminação do ambiente cirúrgico com vapores anestésicos e a necessidade do uso de aparelhagem onerosa limitam uma ampla utilização da anestesia geral inalatória em clínicas veterinárias. Com isso, os profissionais recorrem à anestesia injetável, reduzindo o custo operacional do procedimento cirúrgico.

Uma das técnicas anestésicas injetáveis rotineiramente utilizada por aqueles que não dispõem de um aparelho de anestesia inalatória, é a associação de xilazina e cetamina por via intramuscular (Cullen e Jones, 1977; Haskins et al., 1986; Massone, 1999). A associação destes fármacos por esta via é prática, na medida em que o cirurgião pode efetuar

\footnotetext{
* Departamento de Cirurgia e Anestesiologia Veterinária. FMVZ - UNESP - Campus de Botucatu, SP. stelio@fmvz.unesp.br
} 
a anestesia em dose única (Mohinder et al., 1997; Sharma et al., 1997; Massone, 1999). Adicionalmente, alguns efeitos farmacológicos indesejáveis de uma das substâncias são suplantados pela outra. O relaxamento muscular produzido pela xilazina ameniza a hipertonia muscular causada pela cetamina (Wright, 1982; Pathak et al., 1998). Esta, por sua vez, minimiza os efeitos depressores no sistema cardiovascular (bradicardia, hipotensão e redução do débito cardíaco) e respiratório causados pela xilazina (Kelly et al., 1971; Haskins et al., 1986). Como desvantagem, a associação de xilazina e cetamina causa intenso aumento de secreções, o que pode levar à obstrução respiratória. Com o emprego de substâncias anticolinérgicas na MPA, reduz-se assim a secreção salivar (GomezVillamandos et al., 1998; Massone, 1999).

O objetivo deste trabalho foi investigar o efeito da associação de um agonista $\alpha_{2}$ adrenérgico, a romifidina, com a cetamina na rotina anestésica de cães, comparando-a com o uso clássico da xilazina e cetamina, no intuito de se avaliar um possível aumento do período anestésico, dada a maior duração de ação da romifidina quando comparada à xilazina (England et al., 1992; Lemke, 1999).

\section{Material e métodos}

Foram utilizadas 12 cadelas clinicamente sadias provenientes do Biotério Central da FMVZ-UNESP, Campus de Botucatu, sem raça definida, com peso entre 5 e $19 \mathrm{~kg}$, evitando-se fêmeas em cio e prenhes. Os animais foram submetidos a jejum alimentar de 12 horas e hídrico de seis horas antes da anestesia.

Administrou-se $0,05 \mathrm{mg} / \mathrm{kg}$ de sulfato de atropina ${ }^{1}$ (SC), como medicação pré-anestésica (MPA). Após 15 minutos, seis animais receberam $0,1 \mathrm{mg} / \mathrm{kg}$ de romifidina ${ }^{2}$ associada na mesma seringa a $15 \mathrm{mg} / \mathrm{kg}$ de cetamina ${ }^{3}$ (IM) (ARC) e outros seis, $1 \mathrm{mg} / \mathrm{kg}$ de xilazina ${ }^{4}$ associada na mesma seringa a $15 \mathrm{mg} / \mathrm{kg}$ de cetamina (IM) (AXC).

Os seguintes parâmetros foram avaliados: temperatura retal, ${ }^{5}$ freqüência cardíaca e eletrocardiograma, ${ }^{6}$ pressão sangüínea arterial não-invasiva mensurada na artéria radial, ${ }^{7}$ freqüência respiratória, volume corrente e minuto ${ }^{8}$, concentração de $\mathrm{CO}_{2}$ expirado, saturação de oxigênio nas hemoglobinas, ${ }^{9}$ hemogasometria ( $\mathrm{pH}, \mathrm{PaO}_{2}, \mathrm{PaCO}_{2}$, bicarbonato), hematócrito, osmolaridade sangüínea, concentração sanguínea de sódio, potássio, cloreto, cálcio, glicose e hemoglobina. ${ }^{10} \mathrm{~A}$ colheita de sangue arterial para realização de hemogasometria, quantificação de eletrólitos e glicose foi realizada introduzindo-se agulha hipodérmica $13 \times 4$ na artéria femural. Estas foram colhidas antes da MPA e aos 15 e 45 minutos após a aplicação da romifidina/cetamina ou xilazina/cetamina. Os demais parâmetros foram mensurados 15 minutos antes da MPA, 15 minutos após a MPA e aos 15, 30, 45, 60, 75, 90, 105 e 120 minutos após a administração de romifidina/cetamina ou xiiazina/cetamina.
Avaliou-se o período de latência (da administração dos fármacos agonistas $\alpha 2$-adrenérgicos até a perda do reflexo cutâneo), hábil anestésico (da ausência até o retorno do reflexo cutâneo) e de recuperação anestésica (da administração dos fármacos até a posição quadrupedal).

Adicionalmente, 12 cadelas, sendo seis anestesiadas com $\mathrm{ARC}$ e seis anestesiadas com $\mathrm{AXC}$ foram submetidas à ovariohisterectomia para que o cirurgião avaliasse a adequação da analgesia para a realização do procedimento cirúrgico através do relaxamento de pedículo ovariano.

A análise estatística foi realizada de acordo com Morrison (1967). Utilizando-se análise de variância (ANOVA) para amostras repetidas, seguido pelo teste de Dunnett. As diferenças foram consideradas estatisticamente significantes quando $p<0,05$.

\section{Resultados}

Os períodos de latência, duração e recuperação dos animais submetidos apenas à anestesia, foram de $5 \pm 1,30 \pm 5$ e $55 \pm 5$ minutos para o grupo $A X C$ e $7 \pm 2,75 \pm 12$ e $107 \pm 7$ minutos após a aplicação de $A R C$, sendo estes dois últimos valores significantemente maiores neste último grupo quando comparados ao primeiro. Para os animais submetidos à cirurgia estes foram de $6 \pm 2,83 \pm 28$ e $259 \pm 35$ minutos após $A X C$ e $7 \pm 2,83 \pm 8$ e $411 \pm 150$ minutos após ARC. Adicionalmente, quatro animais do grupo AXC e quatro do grupo ARC necessitaram complementação da anestesia com $5 \mathrm{mg} / \mathrm{kg}$ de cetamina IV e, mesmo assim, em dois dos animais do grupo AXC não foi possível encerrar a cirurgia, sendo necessário o uso de anestesia inalatória com halotano. Foram observados hipertonia muscular e tremores de cabeça dos animais submetidos à cirurgia, em ambos os grupos.

A freqüência cardiaca apresentou um aumento significante apenas após a administração de atropina nos animais do grupo ARC, normalizando em seguida (Quadro 1). Foi observada hipotermia a partir de 45 até 120 minutos em ambos os grupos (Quadro 1). Foram observados redução do $\mathrm{pH}$ e aumento da $\mathrm{PaCO}_{2}$ entre 15 e 45 minutos após romifidina/ cetamina e aos 15 minutos após xilazina/cetamina (Quadro 2). $\mathrm{A} \mathrm{PaO}_{2}$ reduziu aos 45 minutos apenas nos animais tratados com ARC (Quadro 2). Não foi constatada diferença significante para freqüência respiratória, volume minuto, oximetria, concentração de $\mathrm{CO}_{2}$ expirado, pressão arterial média, bicarbonato, hematócrito, concentração sangüínea de sódio, potássio, cloreto, cálcio, glicose e hemoglobina, em relação ao momento basal dentro de cada grupo (Quadros 1 e 2). Observaram-se menores valores de freqüência respiratória (aos 105 minutos), de volume minuto (aos 105 minutos), $\mathrm{pH}$ (aos 45 minutos), pressão arterial (aos 15 e 30 minutos) e maiores valores de cálcio (aos 45 minutos) nos animais tratados com $A R C$, em relação àqueles tratados com $A X C$ (Quadros 1 e 2).

\footnotetext{
1 Sulfato de atropina - Aspen; ${ }^{2}$ Sedivet - Boehringer; ${ }^{3}$ Francotar - Francodex; ${ }^{4}$ Coopazine - Mallinckrodt-Coopers; ${ }^{5}$ Termômetro clínico digital Becton Dickinson; ${ }^{6}$ Funbec 4-1TC/FC; ${ }^{7}$ Dixtal - DX 2710; ${ }^{8}$ Ventilômetro de Wright, Volumeter $3000-$ Drager; $^{9}$ Dixtal DX 7100 ETCO, $/ \mathrm{SpO}_{2} ;{ }^{10} \mathrm{Blood}$ Gas Analyser Stat Profile 5, NOVA Biomedical.
} 
Quadro 1: Freqüência cardíaca, pressão arterial média, hematócrito, concentração sangüínea de sódio, potássio, cálcio, glicose e bicarbonato, hemoglobina e temperatura retal em cães submetidos a anestesia com atropina, romifidina e cetamina - ARC $(n=6)$ e atropina, xilazina e cetamina AXC $(n=6)$.

\begin{tabular}{|c|c|c|c|c|c|c|c|c|c|c|c|}
\hline Minutos & & -15 & 0 & 15 & 30 & 45. & 60 & 75 & 90 & 105 & 120 \\
\hline $\begin{array}{l}\text { FC } \\
\text { (bat/min) }\end{array}$ & $\begin{array}{l}\text { ARC } \\
\text { AXC }\end{array}$ & $\begin{array}{c}106 \pm 8 \\
105 \pm 10\end{array}$ & $\begin{array}{l}159 \pm 25^{*} \\
133 \pm 10\end{array}$ & $\begin{array}{l}129 \pm 14 \\
134 \pm 11\end{array}$ & $\begin{array}{l}142+11 \\
118 \pm 6\end{array}$ & $\begin{array}{c}130 \pm 13 \\
109 \pm 9\end{array}$ & $\begin{array}{l}126 \pm 13 \\
119 \pm 16\end{array}$ & $\begin{array}{c}121 \pm 14 \\
94 \pm 8\end{array}$ & $\begin{array}{c}111 \pm 15 \\
89 \pm 8\end{array}$ & $\begin{array}{l}106 \pm 15 \\
100 \pm 9\end{array}$ & $\begin{array}{l}103 \pm 16 \\
106 \pm 10\end{array}$ \\
\hline $\begin{array}{l}\text { Pressão arterial } \\
(\mathrm{mmHg})\end{array}$ & $\begin{array}{l}\text { ARC } \\
\text { AXC }\end{array}$ & $\begin{array}{l}103 \pm 5 \\
113 \pm 4\end{array}$ & $\begin{array}{c}99 \pm 5 \\
111 \pm 16\end{array}$ & $\begin{array}{c}89 \pm 15 \psi \\
131 \pm 9\end{array}$ & $\begin{array}{l}95 \pm 14 \psi \\
136 \pm 10\end{array}$ & $\begin{array}{l}100 \pm 11 \\
119 \pm 8\end{array}$ & $\begin{array}{c}97 \pm 14 \\
110 \pm 15\end{array}$ & $\begin{array}{c}94 \pm 12 \\
108 \pm 10\end{array}$ & $\begin{array}{l}98 \pm 14 \\
97 \pm 11\end{array}$ & $\begin{array}{r}94 \pm 8 \\
101 \pm 7\end{array}$ & $\begin{array}{c}86 \pm 12 \\
114 \pm 10\end{array}$ \\
\hline $\begin{array}{l}\text { Hematócrito } \\
(\%)\end{array}$ & $\begin{array}{l}\text { ARC } \\
\text { AXC }\end{array}$ & $\begin{array}{l}39 \pm 4 \\
39 \pm 2\end{array}$ & & $\begin{array}{l}38 \pm 4 \\
41 \pm 2\end{array}$ & & $\begin{array}{l}42 \pm 4 \\
42 \pm 2\end{array}$ & & & & & \\
\hline $\begin{array}{l}\text { Sódio } \\
\text { (mmo/L) }\end{array}$ & $\begin{array}{l}\text { ARC } \\
\text { AXC }\end{array}$ & $\begin{array}{l}144 \pm 2 \\
148 \pm 1\end{array}$ & & $\begin{array}{l}147 \pm 2 \\
149+1\end{array}$ & & $\begin{array}{l}146 \pm 1 \\
148 \pm 2\end{array}$ & & & & & \\
\hline $\begin{array}{l}\text { Potássio } \\
\text { (mmol/) }\end{array}$ & $\begin{array}{l}\text { ARC } \\
\text { AXC }\end{array}$ & $\begin{array}{r}3,8 \pm 0,09 \\
4,21 \pm 0,10\end{array}$ & & $\begin{array}{l}3,8 \pm 0,11 \\
4,1 \pm 0,14\end{array}$ & & $\begin{array}{l}4,0 \pm 0,13 \\
4,3 \pm 0,05\end{array}$ & & & & & \\
\hline $\begin{array}{l}\text { Cálcio } \\
\text { (mg/dl) }\end{array}$ & $\begin{array}{l}\text { ARC } \\
\text { AXC }\end{array}$ & $\begin{array}{l}3,37 \pm 0,39 \\
3,82 \pm 0,31\end{array}$ & 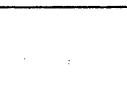 & $\begin{array}{l}4,02+0,16 \\
4,02+0,36\end{array}$ & & $\begin{array}{c}4,16 \pm 0,17 \psi \\
3,17 \pm 0,49\end{array}$ & & & & & \\
\hline $\begin{array}{l}\text { Glicose } \\
\text { (mg/dl) }\end{array}$ & $\begin{array}{l}\text { ARC } \\
\text { AXC }\end{array}$ & $\begin{array}{l}94 \pm 2 \psi \\
111 \pm 4\end{array}$ & & $\begin{array}{l}96 \pm 5 \\
98 \pm 4\end{array}$ & & $\begin{array}{l}105+8 \\
107 \pm 7\end{array}$ & & & & & \\
\hline $\begin{array}{l}\text { Hemoglobina } \\
\text { (g/dl) }\end{array}$ & $\begin{array}{l}\text { ARC } \\
\text { AXC }\end{array}$ & $\begin{array}{l}14 \pm 1 \\
13 \pm 1\end{array}$ & & $\begin{array}{l}13 \pm 1 \\
14 \pm 1\end{array}$ & & $\begin{array}{l}15 \pm 1 \\
14 \pm 1\end{array}$ & & & & & \\
\hline $\begin{array}{l}\text { Bicarbonato } \\
\text { (mmol/ })\end{array}$ & $\begin{array}{l}\text { ARC } \\
\text { AXC }\end{array}$ & $\begin{array}{l}20 \pm 1 \\
22 \pm 1\end{array}$ & & $\begin{array}{l}22 \pm 1 \\
22 \pm 1\end{array}$ & & $\begin{array}{l}22 \pm 1 \\
21 \pm 1\end{array}$ & & & & & \\
\hline $\begin{array}{l}\text { Temperatura } \\
\left({ }^{\circ} \mathrm{C}\right)\end{array}$ & $\begin{array}{l}\text { ARC } \\
\text { AXC }\end{array}$ & $\begin{array}{l}39,0 \pm 0,08 \\
39,4 \pm 0,15\end{array}$ & $\begin{array}{l}39,0 \pm 0,09 \\
39,3 \pm 0,12\end{array}$ & $\begin{array}{l}38,6 \pm 0,12 \\
38,8 \pm 0,36\end{array}$ & $\begin{array}{l}38,2 \pm 0,19 \\
38,4 \pm 0,44\end{array}$ & $\begin{array}{l}37,8 \pm 0,24^{\star} \\
37,7 \pm 0,48^{*}\end{array}$ & $\begin{array}{l}37,5 \pm 0,22^{*} \\
37,6 \pm 0,52^{\star}\end{array}$ & $\begin{array}{l}37,1 \pm 0,32^{*} \\
37,7 \pm 0,60^{*}\end{array}$ & $\begin{array}{l}37,0 \pm 0,29^{\star} \\
37,6 \pm 0,47^{\star}\end{array}$ & $\begin{array}{l}36,8 \pm 0,39^{*} \\
37,5 \pm 0,46^{*}\end{array}$ & $\begin{array}{l}36,8 \pm 0,36^{*} \\
37,6 \pm 0,37^{\star}\end{array}$ \\
\hline
\end{tabular}

* Diferença significante em relação ao basal $(-15 \mathrm{~min})$ ao longo do tempo dentro de cada grupo $(p<0,05)$. $\psi$ Diferença significante entre os grupos em cada momento $(p<0,05)$.

Momentos de avaliação: - 15 - antes da administração de atropina, 0 - após 15 minutos da atropina e antes da anestesia e a seguir a cada 15 minutos da aplicação de romifidina e cetamina ou xilazina e cetamina.

Quadro 2: Freqüência respiratória, volume minuto, $\mathrm{CO}_{2}$ expirado, saturação de $\mathrm{O}_{2}$ nas hemoglobinas, $\mathrm{pH}, \mathrm{PaO}_{2}$ e $\mathrm{PaCO}$ em cães submetidos a anestesia com atropina, romifidina e cetamina - ARC $(n=6)$ e atropina, xilazina e cetamina AXC $(n=6)$.

\begin{tabular}{|c|c|c|c|c|c|c|c|c|c|c|c|}
\hline Minutos & & -15 & 0 & 15 & 30 & 45 & 60 & 75 & 90 & 105 & 120 \\
\hline $\begin{array}{l}\text { FR } \\
\text { (mov/min) }\end{array}$ & $\begin{array}{l}\text { ARC } \\
\text { AXC }\end{array}$ & $\begin{array}{l}19 \pm 2 \\
25 \pm 5\end{array}$ & $\begin{array}{l}20 \pm 4 \\
21 \pm 4\end{array}$ & $\begin{array}{l}17 \pm 5 \\
20 \pm 4\end{array}$ & $\begin{array}{l}16 \pm 2 \\
21 \pm 4\end{array}$ & $\begin{array}{l}16 \pm 3 \\
23 \pm 5\end{array}$ & $\begin{array}{l}16 \pm 3 \\
25 \pm 5\end{array}$ & $\begin{array}{l}14 \pm 3 \\
20 \pm 4\end{array}$ & $\begin{array}{l}13 \pm 2 \\
20 \pm 5\end{array}$ & $\begin{array}{c}11 \pm 2 \psi \\
18 \pm 2\end{array}$ & $\begin{array}{l}11 \pm 2 \\
14 \pm 1\end{array}$ \\
\hline $\begin{array}{l}\text { Volume minuto } \\
\text { (L) }\end{array}$ & $\begin{array}{l}\text { ARC } \\
\text { AXC }\end{array}$ & $\begin{array}{l}21,4 \pm 3,4 \\
35,0 \pm 6,4\end{array}$ & $\begin{array}{l}18,8 \pm 1,8 \\
34,8 \pm 7,0\end{array}$ & $\begin{array}{l}10,0 \pm 1,1 \\
16,0 \pm 2,7\end{array}$ & $\begin{array}{l}19,2 \pm 5,3 \\
21,8 \pm 2,7\end{array}$ & $\begin{array}{l}20,3 \pm 4,2 \\
22,7 \pm 5,4\end{array}$ & $\begin{array}{l}22,8 \pm 6,1 \\
21,0 \pm 2,5\end{array}$ & $\begin{array}{l}20,0 \pm 6,6 \\
24,5 \pm 3,1\end{array}$ & $\begin{array}{l}19,5 \pm 5,4 \\
21,7 \pm 1,3\end{array}$ & $\begin{array}{c}17,0 \pm 3,2 \psi \\
27,3 \pm 2,6\end{array}$ & $\begin{array}{l}17,4 \pm 3,7 \\
26,3 \pm 1,6\end{array}$ \\
\hline $\begin{array}{l}\mathrm{CO}_{2} \text { expirado } \\
(\mathrm{mmHg})\end{array}$ & $\begin{array}{l}\text { ARC } \\
\text { AXC }\end{array}$ & $\begin{array}{l}41 \pm 1 \\
40 \pm 2\end{array}$ & $\begin{array}{l}42 \pm 2 \\
39 \pm 2\end{array}$ & $\begin{array}{l}44 \pm 2 \\
41 \pm 3\end{array}$ & $\begin{array}{l}43 \pm 2 \\
39 \pm 1\end{array}$ & $\begin{array}{l}38 \pm 3 \\
36 \pm 2\end{array}$ & $\begin{array}{l}36 \pm 2 \\
31 \pm 3\end{array}$ & $\begin{array}{l}38 \pm 2 \\
31 \pm 4\end{array}$ & $\begin{array}{l}35 \pm 3 \\
37 \pm 2\end{array}$ & $\begin{array}{l}39 \pm 1 \\
39 \pm 1\end{array}$ & $\begin{array}{l}41 \pm 1 \\
41 \pm 2\end{array}$ \\
\hline $\begin{array}{l}\text { Saturação de } \mathrm{O}_{2} \\
(\%)\end{array}$ & $\begin{array}{l}\text { ARC } \\
\text { AXC }\end{array}$ & $\begin{array}{l}92 \pm 1 \\
93 \pm 3\end{array}$ & $\begin{array}{l}94 \pm 1 \\
91 \pm 3\end{array}$ & $\begin{array}{l}92 \pm 1 \\
95 \pm 1\end{array}$ & $\begin{array}{l}92 \pm 1 \\
94 \pm 1\end{array}$ & $\begin{array}{l}92 \pm 1 \\
92 \pm 1\end{array}$ & $\begin{array}{l}91 \pm 3 \\
88 \pm 2\end{array}$ & $\begin{array}{l}88 \pm 2 \\
90 \pm 1\end{array}$ & $\begin{array}{l}93 \pm 1 \\
93 \pm 1\end{array}$ & $\begin{array}{l}91 \pm 1 \\
93 \pm 1\end{array}$ & $\begin{array}{l}93 \pm 1 \\
93 \pm 1\end{array}$ \\
\hline $\mathrm{pH}$ & $\begin{array}{l}\text { ARC } \\
\text { AXC }\end{array}$ & $\begin{array}{l}7,45 \pm 0,01 \\
7,43 \pm 0,01\end{array}$ & & $\begin{array}{l}7,37 \pm 0,01^{*} \\
7,39 \pm 0,01^{*}\end{array}$ & & $\begin{array}{l}7,37 \pm 0,01^{*} \\
7,41 \pm 0,01 \psi\end{array}$ & & & & & \\
\hline $\begin{array}{l}\mathrm{PaO}_{2} \\
(\mathrm{mmHg})\end{array}$ & $\begin{array}{l}\text { ARC } \\
\text { AXC }\end{array}$ & $\begin{array}{l}92 \pm 3 \\
88 \pm 5\end{array}$ & & $\begin{array}{l}79 \pm 5 \\
73 \pm 6\end{array}$ & & $\begin{array}{l}70 \pm 6^{*} \\
90 \pm 6\end{array}$ & & & & & \\
\hline $\begin{array}{l}\mathrm{PaCO}_{2} \\
(\mathrm{mmHg})\end{array}$ & $\begin{array}{l}\text { ARC } \\
\text { AXC }\end{array}$ & $\begin{array}{l}28 \pm 1 \\
32 \pm 2\end{array}$ & & $\begin{array}{l}38 \pm 2^{*} \\
36 \pm 1^{*}\end{array}$ & & $\begin{array}{l}38 \pm 2^{*} \\
32 \pm 1\end{array}$ & & & & & \\
\hline
\end{tabular}

* Diferença significante em relação ao basal $(-15 \mathrm{~min})$ ao longo do tempo dentro de cada grupo $(p<0,05)$.

$\psi$ Diferença significante entre os grupos em cada momento $(p<0,05)$.

Momentos de avaliação: -15 - antes da administração de atropina, 0 - após 15 minutos da atropina e antes da anestesia e a seguir a cada 15 minutos da aplicação de romifidina e cetamina ou xilazina e cetamina. 


\section{Discussão}

A maior duração anestésica produzida pela associação de $A R C$, quando comparada à $A X C$, corroborou com informações obtidas por England et al. (1992). A recuperação foi mais prolongada quando se utilizou romifidina, bem como mais prolongada quando os animais foram submetidos à cirurgia, demonstrando que o estresse produzido por este procedimento cruento pode aumentar o tempo de recuperação. Adicionalmente, nos animais submetidos à cirurgia, complementou-se a anestesia o que, provavelmente, prolongou o período de recuperação.

A taquicardia observada após a administração de atropina é um efeito típico do fármaco, devido ao seu efeito anticolinérgico (Rishniw et al., 1996; Gomez-Villamandos et al., 1998; Massone, 1999; Rishniw et al., 1999). Deste modo, a atropina efetivamente diminuiu a sialorréia e estabilizou a freqüência cardíaca, evitando bradicardia e arritmias normalmente observadas com o uso de agonistas $\alpha_{2-}$ adrenérgicos. Portanto, a mesma é indicada como MPA, quando se utilizam fármacos desta natureza (Alibhai et al., 1996; Massone, 1999).

O mesmo foi observado em relação à pressão arterial, que permaneceu estável durante a anestesia. Os agonistas $\alpha_{2}$. adrenérgicos normalmente causam um período de hipertensão inicial, por estímulo $a_{2}$ pós-sináptico, seguido de hipotensão (Klide, 1975). Provavelmente esta rápida fase de hipertensão não foi detectada no experimento, pois os fármacos foram administrados por via intramuscular e a primeira mensuração foi realizada apenas 15 minutos após a administração de romifidina ou xilazina. A cetamina provavelmente evitou a hipotensão arterial normalmente produzida pela romifidina e xilazina (Clarke et al., 1991; Alibhai et al., 1996; Massone, 1999).

\section{Referências}

ALIBHAI, H.I.K.; CLARKE. K.W.; LEE,Y.H.; THOMPSON, J. Cardiopulmonary effects of combinations of medetomidine hydrochloride and atropine sulphate in dogs. Vet. Record., v. 138, n. 1, p. 11-13, 1996.

AMRESH, K.; BHARAT,S.; KUMAR,A.; SINGH,B. Effects of mixture of diazepam and xylazine in dogs. Ind. J. Vet. Sur., v. 15, n. 1, p. 7-10, 1994.

CLARKE, K.W.; ENGLAND,G.C.W.; GOOSSENS,L. Sedative and cardiovascular effects of romifidine alone and in combination with butorphanol, in the horse. J. Vet. Anaesth., v. 18, p. 25-29, 1991.

CULLEN, L.K.; JONES,R.S. Clinical observations on xylazine/ketamine anaesthesia in the cat. Vet. Record., v. 101, p. 115-116, 1977.

ENGLAND, G.C.W; CLARKE, K.W.; GOOSSENS, L. A comparison of the sedative effects of three alpha ${ }_{2}$-adrenoceptor agonists (romifidine, detomidine and xylazine) in the horse. J. Vet. Pharmacol. Therap., v. 15, p. 194-201, 1992.

GASTHUYS, F.; TERPSTRA, P.; VAN DAN HENDE, C.; MOOR, A. Hyperglycaemia and diuresis during sedation with detomidine in the horse. J. Vet. Med. Assoc., v. 34, p. 641-648, 1987.

GOMEZ-VILLAMANDOS, R.J.; SANTISTEBAN, J.M.; REDONDO, J.I.; DOMINGUEZ, J.M.; GALKA, M.E.; RUIZ,I.; AVILA, I. Anticholinergic drugs in veterinary anaesthesia. Med. Veterinaria, v. 15, n. 4, p. $197-$ 211, 1998.

HASKINS, S.C.; PATZ, J.D.; FARVER, T.B. Xylazine and xylazineketamine in dogs. Am. J. Vet. Res., v. 47, p. 636-641, 1986.
A hipotermia foi produzida pelos fármacos utilizados durante a anestesia e provavelmente deveu-se a vasodilatação periférica, depressão do centro termorregulador, redução do metabolismo basal, redução da produção de calor e aumento da perda de calor pela via respiratória (Scheidy, 1959; Short, 1987).

A discreta redução do $\mathrm{pH}$ e aumento da $\mathrm{PaCO}_{2}$ deveu-se possivelmente à depressão respiratória ocasionada pelos agonistas $\alpha_{2}$ adrenérgicos (Haskins et al., 1986; Amresh et al., 1994) já que a cetamina aumenta a resposta respiratória em cães tanto normo como hipercapnéicos, causando uma elevação do volume minuto, sendo, portanto, improvável que tenha contribuído para esta depressão (Kelly et al., 1971). Observou-se maior depressão cardiorrespiratória no grupo tratado com ARC, caracterizada por bradipnéia, diminuição do volume minuto, $\mathrm{pH}$, pressão arterial, bem como redução da $\mathrm{PaO}_{2}$, idêntica às observações de Clarke et al. (1991) na espécie eqüina. Apesar destas alterações, os parâmetros permaneceram próximos à faixa de normalidade, demonstrando estabilidade proporcionada pela associação dos fármacos utilizados neste grupo.

A ausência de alterações eletrolíticas demonstra que a associação de ARC e AXC não alterara a fração metabólica do equilibrio ácido-básico, nem as trocas iônicas entre o líqüido extra e intracelular. Os agonistas $\alpha_{2}$ adrenérgicos normalmente aumentam a concentração de glicose devido ao efeito antiinsulina e/ou inibição da oxidação da glicose (Gasthuys et al., 1987). Apesar do discreto aumento observado neste estudo, as alterações não foram significantes.

Concluiu-se que, apesar das técnicas anestésicas empregadas serem seguras sob o ponto de vista cardiorrespiratório, o fato da analgesia cirúrgica ter sido insuficiente na maioria dos animais sugere que nenhuma destas associações nas doses empregadas neste estudo é indicada para a realização de ovario-histerectomia em cadelas.

KELLY, R.W.; WILSON,R.D.; TRABER,D.L.; PRIANO,L.L. Effects of two new dissociative anesthetic agents, ketamine and $\mathrm{CL}-1848 \mathrm{C}$, on the respiratory response to carbon dioxide. Anesth. Analg., v. 50, p. 262$269,1971$.

KLIDE, A.M.; CALDERWOOD,H.W.; SOMA,L.R. Cardiopulmonary effects of xylazine in dogs. Am. J. Vet. Res., v. 36, p. 931-935, 1975.

LEMKE, K.A. Sedative effects of intramuscular administration of a low dose of romifidine in dogs. Am. J. Vet. Res., v. 60, n. 2, p. 162-168, 1999.

MASSONE, F. Anestesioiogia Veterinána 3. ed. Rio de Janeiro: Guanabara Koogan, 1999.

MOHINDER, S.; NIGAM,J.M.; MADHUMEET,S.; VINEY,K.; SHARMA,S.K.; VARSHNEY, A.O.; SINGH,M.; KUMAR, V. Clinical evaluation of xylazine and ketamine combination in canines. Ind. Vet. Journal., v. 74, n. 4, p. 354-355, 1997.

MORRISON, D.F. Multivariate Statistical Methods. New York: McGraw Hill, 1967.

PATHAK, J.O.; PANDEY,S.S.; SHUKLA,B.P.; SINGH,V. Clinical evaluation of xylazine with barbiturate and non-barbiturate anaesthesia in dogs. Ind. Vet. Journal., v. 75, n. 10, p. 895-898, 1998.

RISHNIW, M.; KITTLESON,M.D.; JAFFE,R.S.; KASS,P.H. Characterization of parassympatholytic chronotropic responses following intravenous administration of atropine to clinically normal dogs. Am. J. Vet. Res., v. 60, n. 8, p. 1000-1003, 1999.

RISHNIW, M.; TOBIAS,A.H.; SLINKER,B.K. Characterization of chronotropic and dysrhythmogenic effects of atropine in dogs with bradycardia. Am J. Vet. Res, v. 57, n. 3, p. 337-341, 1996. 
SCHEIDY, S.F. Brief review of tranquilizing drugs in veterinary practice. The Swest. Vet., v. 2, p. 271-274. 1959.

SHARMA, S.K.;MOHINDER,S.; VARSHNEY.A.O.; VINAY,K;; SURINDER. G.; NIGAM, J.M.; SINGH, M.; KUMAR, V. GUPTA, S. Atropine-xylazineketamine as balanced anaesthesia for canines - a clinical study. Ind. Vet. Jour., v. 74. n. 7, p. 613-615, 1997.
SHORT, C.E. Anticholinergics. In: SHORT,C.E. (ed). Principles and Practice of Veterinary Anesthesia. Baltimore: Williams \& Wilkins, 1987. p. 8-15.

WRIGHT, M. Pharmacologic effects of ketamine and its use in veterinary medicine. J. Am. Vet. Med. Assoc., v. 180, p. 1462-1471, 1982. 